\title{
Macrocephaly in neurofibromatosis type 1: a sign post for optic pathway gliomas?
}

\author{
Christina Schindera • Kevin Wingeier • Barbara Goeggel Simonetti • Miriam Diepold • \\ Claude B. Nauer • Johannes Fleischhauer • Maja Steinlin
}

Received: 28 July 2011 / Accepted: 29 July 2011 / Published online: 7 August 2011

(C) Springer-Verlag 2011

\begin{abstract}
Purpose Optic pathway gliomas, which occur in 15-20\% of paediatric patients with neurofibromatosis type 1 , are the most common central nervous system tumour associated with this neurocutaneous disorder. The detection of optic pathway gliomas is essential for further management but is often delayed in infancy due to oligosymptomatic progression and difficulties in clinical detection. Therefore, the aim of our study was to find a clinical indicator for the presence of optic pathway gliomas in children with neurofibromatosis type 1 in order to facilitate early diagnosis and initiate further ophthalmological and neuroimaging investigations. Methods We retrospectively evaluated 70 patients (mean age of 10.5 years; SD of 4.3 years; range of $0.5-19.6$ years;
\end{abstract}

C. Schindera $(\bowtie) \cdot$ K. Wingeier $\cdot$ B. Goeggel Simonetti $\cdot$

M. Steinlin

Paediatric Neurology, University Children's Hospital, Inselspital, 3010 Bern, Switzerland

e-mail: christina.schindera@gmx.de

K. Wingeier

Division of Experimental Psychology and Neuropsychology,

University of Bern,

Bern, Switzerland

M. Diepold

Paediatric Haematology and Oncology, University Children's

Hospital, Inselspital,

Bern, Switzerland

C. B. Nauer

Diagnostic and Interventional Neuroradiology, University

Hospital, Inselspital,

Bern, Switzerland

J. Fleischhauer

Ophthalmology, University Hospital, Inselspital,

Bern, Switzerland
35 females) with neurofibromatosis type 1 seen at the University Children's Hospital of Bern, Switzerland, between January 1998 and December 2008 regarding clinical features of neurofibromatosis type 1 in relation to the presence of optic pathway gliomas.

Results Fifty-seven of the 70 patients (81.4\%) had no clinical or radiological signs of optic pathway gliomas [magnetic resonance imaging (MRI) of the brain in 26/57], whereas 13/70 patients (18.6\%) were diagnosed with optic pathway gliomas by MRI. Patients with optic pathway gliomas showed macrocephaly significantly more often compared to patients without optic pathway gliomas $(8 / 13$ vs. $9 / 57$, respectively; $p=0.004$ ).

Conclusion Macrocephaly significantly correlates with the incidence of optic pathway gliomas in children with neurofibromatosis type 1 . We therefore hypothesise that in otherwise asymptomatic patients, macrocephaly is an additional indicator for performing MRI to detect optic pathway gliomas.

Keywords Neurofibromatosis-type 1 . Optic pathway gliomas · Macrocephaly · Children · MRI

\section{Introduction}

Neurofibromatosis type 1 (NF1) is an autosomal dominant neurocutaneous genetic disorder occurring with an estimated incidence of 1:3,000 individuals per year, independent of ethnicity, race and gender [1]. The hallmarks of the clinical condition are the development of pigmentary lesions (caféau-lait spots, skinfold freckling and Lisch nodules), distinctive skeletal lesions (sphenoid dysplasia and pseudoarthrosis) and tumours, such as optic pathway gliomas and neurofibromas [2]. 
Table 1 Results of the Barnard's test: comparison of group 1 (NF1 without OPGs) and group 2 (NF1 with OPGs) patients regarding NIH criteria
OPGs optic pathway gliomas, NF1 neurofibromatosis type 1

\begin{tabular}{llll}
\hline & $\begin{array}{l}\text { Group 1 patients (NF1 without } \\
\text { OPGs) } n=57 / 70(81.4 \%)\end{array}$ & $\begin{array}{l}\text { Group 2 patients: (NF1 with } \\
\text { OPGs) } n=13 / 70(18.6 \%)\end{array}$ & $p$ value \\
\hline $\begin{array}{l}\text { Mean age, in years, at } \\
\text { last consultation [SD] }\end{array}$ & $10.6[4.6]$ & $10.1[3.4]$ & 0.733 \\
$\begin{array}{l}\text { (Student's } t \text { test) } \\
\text { Café-au-lait spots }\end{array}$ & $56 / 57(98.3 \%)$ & & \\
Skinfold freckling & $39 / 57(68.4 \%)$ & $13 / 13(100 \%)$ & 1.000 \\
Lisch nodules & $3 / 57(5.3 \%)$ & $12 / 13(92.3 \%)$ & 0.167 \\
Skeletal dysplasia & $7 / 57(12.3 \%)$ & $2 / 13(15.4 \%)$ & 0.255 \\
Neurofibromas & $20 / 57(35.1 \%)$ & $2 / 13(15.4 \%)$ & 1.000 \\
Positive family history & $24 / 57(42.1 \%)$ & $4 / 13(38.5 \%)$ & 1.000 \\
\hline
\end{tabular}

The NF1 gene product, neurofibromin, is known to act as a tumour suppressor [3]. Loss of neurofibromin leads to increased activity of the Ras signalling pathway and therefore to accelerated cell growth with astrocyte proliferation [3, 4]. However, in the developing brain, the NF1 gene also functions as a cell growth regulator and seems to play a role in neuronal differentiation [5]. Disturbed cell growth regulation might be the backbone of an abnormal brain development, as seen in the $20 \%$ rate of learning disabilities in paediatric NF1 patients [6].

Optic pathway gliomas (OPGs) are the predominant cerebral tumour in NF1, affecting $15-20 \%$ of all children with NF1 within their first decade of life [7]. Histologically, OPGs are almost uniformly pilocytic astrocytomas (WHO grade I). Many of these tumours show prolonged indolent phases, whereas others progress rapidly. In addition, OPGs can have erratic growth patterns or may demonstrate spontaneous regression [8, 9]. However, only $30-50 \%$ of children with NF1 and OPGs develop clinical symptoms such as decreased visual acuity, proptosis or precocious puberty, thus making an early diagnosis difficult [10].

In an attempt to facilitate early diagnosis of OPGs in asymptomatic and oligosymptomatic children with NF1, the present study analysed clinical characteristics such as macrocephaly, epilepsy, headache/migraine, scoliosis, neurocognitive deficits and behavioural abnormalities in order to determine additional indicators for early ophthalmologic and neuroimaging investigations.

\section{Materials and methods}

This study complied with Health Insurance Portability and Accountability Act and was approved by our institutional review board. In addition, the requirement for an informed consent was waived. The current study is a retrospective review of all consecutive paediatric and adolescent patients with NF1 referred to the University Children's Hospital in Bern, Switzerland, between 1 January 1998 and 31 December 2008. On a routine basis, these children were seen over a yearly schedule by a neuropaediatrician and an ophthalmologist. Study enrolment required that the subjects fulfilled the diagnostic criteria of the 1988 National Institutes of Health (NIH) Consensus Development Conference for Neurofibromatosis Type 1 [2]. Patient charts and radiology records were reviewed for data relating to patient demographics, the clinical distribution of NIH criteria (café-au-lait spots, skinfold freckling, Lisch nodules, skeletal dysplasia, neurofibromas, optic pathway gliomas and a first-degree relative who was positive for NF1) and the presence of NF1-related neurological abnormalities (macrocephaly, epilepsy, headache/migraine, scoliosis and neurocognitive deficits such as learning deficits and behavioural abnormalities).

Patients were further divided into two groups: children with NF1 without OPGs (group 1) and children with NF1 and the presence of OPGs (group 2). OPGs were solely diagnosed by magnetic resonance imaging (MRI) of the brain. Imaging techniques were performed by clinical indication in children
Table 2 Results of the Barnard's test: comparison of group 1 (NF1 without OPGs) and group 2 (NF1 with OPGs) patients regarding NF1-associated features and the presence of unknown bright objects

$O P G s$ optic pathway gliomas, NF1 neurofibromatosis type 1

${ }^{\text {a }}$ Statistically significant

\begin{tabular}{lccc}
\hline & $\begin{array}{c}\text { Group 1 patients (NF1 } \\
\text { without OPGs) } n=57(81.4 \%)\end{array}$ & $\begin{array}{l}\text { Group 2 patients (NF1 } \\
\text { with OPGs) } n=13(18.6 \%)\end{array}$ & $p$ value \\
\hline Macrocephaly & $9 / 57(15.8 \%)$ & $8 / 13(61.5 \%)$ & $0.004^{\mathrm{a}}$ \\
Epilepsy & $2 / 57(3.5 \%)$ & $2 / 13(15.4 \%)$ & 0.183 \\
Headache/migraine & $10 / 57(17.5 \%)$ & $0 / 13(0 \%)$ & 0.183 \\
Scoliosis & $22 / 57(38.6 \%)$ & $5 / 13(38.5 \%)$ & 1.000 \\
Learning disabilities & $19 / 57(33.3 \%)$ & $6 / 13(46.2 \%)$ & 0.472 \\
Behavioural abnormalities & $17 / 57(29.8 \%)$ & $2 / 13(15.4 \%)$ & 0.367 \\
Unknown bright objects (UBOs) & $15 / 26(57.7 \%)$ & $11 / 13(84.6 \%)$ & 0.120 \\
\hline
\end{tabular}


Table 3 Results of the Barnard's test: comparison of group 1 (NF1 without OPGs) and group 2 (NF1 with OPGs) patients with and without macrocephaly regarding the performance of an MRI of the brain and the presence of unknown bright objects

\begin{tabular}{|c|c|c|c|c|c|c|}
\hline & $\begin{array}{l}\text { Group } 1 \text { patients } \\
\text { (NF1 without OPGs) }\end{array}$ & & $p$ value & $\begin{array}{l}\text { Group } 2 \text { patients } \\
\text { (NF1 with OPGs) }\end{array}$ & & $p$ value \\
\hline & $\begin{array}{l}\text { With macrocephaly } \\
n=9 / 57(15.8 \%)\end{array}$ & $\begin{array}{c}\text { Without macrocephaly } \\
n=48 / 57(84.2 \%)\end{array}$ & & $\begin{array}{c}\text { With macrocephaly } \\
n=8 / 13(61.5 \%)\end{array}$ & $\begin{array}{c}\text { Without macrocephaly } \\
n=5 / 13(38.5 \%)\end{array}$ & \\
\hline MRI of the brain & $2 / 9(7.7 \%)$ & $24 / 48(54.2 \%)$ & 0.189 & $8 / 8(61.5 \%)$ & $5 / 5(38.5 \%)$ & 1.000 \\
\hline $\begin{array}{l}\text { Unknown bright objects } \\
\text { (UBOs) }\end{array}$ & $2 / 2(100 \%)$ & $13 / 24(54.2 \%)$ & 0.365 & $7 / 8(87.5 \%)$ & $4 / 5(80.0 \%)$ & 0.849 \\
\hline
\end{tabular}

OPGs optic pathway gliomas, NF1 neurofibromatosis type 1, MRI magnetic resonance imaging

with symptoms or medical findings in a routine examination that required further investigation (e.g. headache, neurological abnormalities or decreased visual acuity). All images were reviewed at the time of imaging by an experienced neuroradiologist for the presence of OPGs or other brain tumours. At the time of study accomplishment, the images were re-evaluated regarding a ventricle/parenchyma ratio by measuring the cross diameter of the third ventricle at the level of the thalami and the cross diameter of the parenchyma at the level of the foramina of Monro to differentiate hydrocephalus from megalencephaly. Furthermore, tumours were classified radiologically according to the number and location. Diagnosed patients with OPG were further evaluated regarding symptoms related to $\mathrm{OPG}$ and the initiated therapy.

Using the Student's $t$ test for the ventricle/parenchyma ratio and the Barnard's test for the remaining calculations, we calculated possible differences among the variables between the two patient groups [11]. The null hypothesis was that both groups are equal. The hypothesis was rejected if $p<0.05$ in a two-tailed analysis (Tables 1, 2 and 3).

\section{Results}

During the 10-year study period, 70 children [mean age at last follow-up of 10.5 years; standard deviation (SD) of 4.3 years; range of 0.5-19.6 years; 35 females] with NF1 were referred to the University Children's Hospital in Bern. MRI was performed in $55.7 \%(39 / 70)$ of the participating patients, broken down as $45.6 \%(26 / 57)$ of group 1 patients and all patients of group $2(13 / 13)$. Fifty-seven of the 70
(81.4\%) patients had no clinical or radiological evidence of OPGs (group 1, mean age at last presentation of 10.6 years; SD of 4.6 years; 29 females), whereas 13/70 (18.6\%) patients were diagnosed with OPG by MRI of the brain (group 2, mean age at last presentation of 10.1 years; SD of 3.4 years; six females) (Table 1). There was no significant difference between the groups regarding the distribution of NIH criteria, except for the presence of OPGs (Table 1).

However, $15.8 \%$ of group 1 patients showed macrocephaly compared to $61.5 \%$ of group 2 patients. This difference was statistically significant $(p=0.004)$ (Table 2). In group 1 patients with macrocephaly, two of nine $(7.7 \%)$ had an MRI of the brain, whereas 24/48 (54.2\%) group 1 patients without macrocephaly had an MRI of the brain ( $p=$ 0.189) (Table 3). Limiting the number to children who have had MRI of the brain shows that 2/26 (7.7\%) group 1 patients had macrocephaly, whereas $8 / 13$ (61.5\%) group 2 patients had macrocephaly. This difference was still statistically significant $(p=0.0001)$ (Table 4). Evaluating the ventricle/parenchyma ratio at the level of the third ventricle of group 1 (measured in 21/26) and group 2 (measured in 11/13) patients showed no statistical significance $(0.22$ vs. $0.26 ; p=0.620)$. In two of two $(100 \%)$ group 1 patients with macrocephaly, unknown bright objects (UBOs) were identified on the MRI of the brain, while only $13 / 24(54.2 \%)$ group 1 patients without macrocephaly had UBOs on the MRI of the brain $(p=0.365)$ (Table 3 ). Group 2 patients with and without macrocephaly did not show any differences regarding the presence of UBOs (87.5\% vs. $80.0 \% ; p=0.849$ ) (Table 3 ). In addition, there were no statistically significant differences regarding

Table 4 Results of the Barnard's test: comparison of group 1 (NF1 without OPGs) and group 2 (NF1 with OPGs) patients with both macrocephaly and MRI of the brain

Group 1 patients (NF1 without OPGs) with MRI of the brain $n=26$
Group 2 patients (NF1 with OPGs) with MRI of the brain $n=13$ $p$ value

$8 / 13(61.5 \%)$

$0.0001^{\mathrm{a}}$ 
epilepsy, headache/migraine, scoliosis, learning disabilities and behavioural abnormalities (Table 2).

Regarding the presence of OPGs, 10/13 (76.9\%) group 2 patients had chiasmal OPGs, whereas 4/13 (30.7\%) had OPGs involving one optic nerve or both optic nerves, separately, and 2/13 (15.4\%) group 2 patients showed OPGs affecting the optic tract. Six of $13(46.2 \%)$ patients initially presented with visual impairment, and 2/13 (15.4\%) showed elevated intracranial pressure due to additional cerebral gliomas. However, 5/13 (38.5\%) patients of group 2 were asymptomatic.

Chemotherapeutic treatment with drugs (carboplatin, etoposide and temozolamide) was started in 3/13 (23.1\%) patients due to progressive visual impairment. These patients were between 9 and 10 years of age at the last follow-up, and all had significant visual impairment (visual acuity $<0.3$ ). The remaining patients showed no, or mild, visual impairment.

\section{Discussion}

The aim of our study was to find besides visual problems a clinical indicator for the presence of OPGs in paediatric patients with NF1 in order to facilitate early diagnosis and to initiate MRI of the brain as early as necessary. Our results show that macrocephaly correlates significantly with the presence of OPGs in paediatric patients with NF1, whom are also otherwise asymptomatic patients. Macrocephaly, defined as a head circumference two or more standard deviations above the sex- and age-matched population's mean, has clearly been associated with NF1, occurring in $25-50 \%$ of all patients with NF1 [12-14]. Macrocephaly was revealed in $24 \%$ of patients in our cohort. However, $61.5 \%$ of NF1 patients with OPGs demonstrated macrocephaly, whereas only $15.8 \%$ of NF1 patients without OPGs had macrocephaly. Neuroimaging of all the patients might have increased the number of patients with OPG. However, our results were still significant limiting the number to children who have had MRI of the brain. The likelihood of developing OPG after normal MRI of the brain and careful clinical and ophthalmological follow-up must be considered minimal.

In NF1, macrocephaly is caused by megalencephaly, which is defined as the enlargement of brain tissue [12]. This enlargement is mainly due to a significant increase in white matter volume when compared to normocephalic NF1 patients [12]. In addition to brain enlargement, 60$70 \%$ of NF1 patients show so-called UBOs in the brain tissue that are characteristic focal lesions of increased signal intensity on T2-weighted MRIs [14, 15]. These lesions reflect dysplastic glial proliferations in the developing brain $[15,16]$ and are significantly related to lower IQs in NF1- affected children $[15,17]$. Thus far, only Steen et al. could demonstrate a correlation between the presence of bilateral UBOs and macrocephaly in paediatric patients with NF1 [12]. In the present study, UBOs were more frequently seen in patients with than without OPGs, but this difference was not statistically significant because of the small patient number. In summary, these NF1-specific alterations suggest that children with macrocephaly, UBOs and OPGs might have lower levels of neurofibromin and therefore more pronounced accelerated cell growth. Moreover, Szudek et al. studied statistical associations among 13 of the most important and common clinical features in NF1 [18]. It was reported that macrocephaly and OPGs have strong associations, suggesting that glial hyperplasia is responsible not only for macrocephaly but also for the development of optic pathway gliomas [13, 18, 19].

Currently, routine neuroimaging screening for OPGs in asymptomatic paediatric patients is not recommended [20]. However, children younger than 6 years of age are at the greatest risk of OPG development [7]. In this age group, the expression of decreased visual acuity, as the most common clinical symptom in patients with OPGs, is unreliable [20].

Limitations of our study must be acknowledged. Data were collected retrospectively, and imaging techniques were not performed in all 70 patients. Therefore, it might be possible that some undiagnosed patients with OPGs were false subjects of group 1. Furthermore, the number of participants in the present study was quite small, so larger studies are necessary to confirm our findings. We conclude that macrocephaly may be useful as a quick and inexpensive indicator for performing an MRI of the brain or thorough ophthalmological investigations in otherwise asymptomatic paediatric patients with NF1 to scan for OPGs.

Acknowledgments The authors would like to thank all of the children and adolescents whose data were used for the analysis.

Conflict of interest The authors declare that they have no conflict of interest.

\section{References}

1. Friedman JMRV (1999) Clinical and epidemiological features. Neurofibromatosis: phenotype, natural history, and pathogenesis. John Hopkins University Press, Baltimore, pp 29-86

2. National Institutes of Health Consensus Development Conference Statement: neurofibromatosis (1988) Bethesda, MD, USA, July 13-15, 1987. Neurofibromatosis 1: 172-178

3. Basu TN, Gutmann DH, Fletcher JA, Glover TW, Collins FS, Downward J (1992) Aberrant regulation of ras proteins in malignant tumour cells from type 1 neurofibromatosis patients. Nature 356:713-715

4. Lau N, Feldkamp MM, Roncari L, Loehr AH, Shannon P, Gutmann DH, Guha A (2000) Loss of neurofibromin is associated 
with activation of RAS/MAPK and PI3-K/AKT signaling in a neurofibromatosis 1 astrocytoma. J Neuropathol Exp Neurol 59:759-767

5. Giordano MJ, Mahadeo DK, He YY, Geist RT, Hsu C, Gutmann DH (1996) Increased expression of the neurofibromatosis 1 (NF1) gene product, neurofibromin, in astrocytes in response to cerebral ischemia. J Neurosci Res 43:246-253

6. Hyman SL, Shores A, North KN (2005) The nature and frequency of cognitive deficits in children with neurofibromatosis type 1 . Neurology 65:1037-1044

7. Listernick R, Charrow J, Greenwald M, Mets M (1994) Natural history of optic pathway tumors in children with neurofibromatosis type 1: a longitudinal study. J Pediatr 125:63-66

8. Opocher E, Kremer LC, Da Dalt L, van de Wetering MD, Viscardi E, Caron HN, Perilongo G (2006) Prognostic factors for progression of childhood optic pathway glioma: a systematic review. Eur J Cancer 42:1807-1816

9. Perilongo G, Moras P, Carollo C, Battistella A, Clementi M, Laverda A, Murgia A (1999) Spontaneous partial regression of low-grade glioma in children with neurofibromatosis-1: a real possibility. J Child Neurol 14:352-356

10. Listernick R, Darling C, Greenwald M, Strauss L, Charrow J (1995) Optic pathway tumors in children: the effect of neurofibromatosis type 1 on clinical manifestations and natural history. $\mathrm{J}$ Pediatr 127:718-722

11. Barnard GA (1945) A new test for $2 \times 2$ tables. Nature 156:177

12. Steen RG, Taylor JS, Langston JW, Glass JO, Brewer VR, Reddick WE, Mages R, Pivnick EK (2001) Prospective evaluation of the brain in asymptomatic children with neurofibromatosis type 1: relationship of macrocephaly to $\mathrm{T} 1$ relaxation changes and structural brain abnormalities. AJNR Am J Neuroradiol 22:810 817

13. Szudek J, Birch P, Friedman JM (2000) Growth in North American white children with neurofibromatosis 1 (NF1). J Med Genet 37:933-938

14. Van Es S, North KN, McHugh K, De Silva M (1996) MRI findings in children with neurofibromatosis type 1: a prospective study. Pediatr Radiol 26:478-487

15. North K, Joy P, Yuille D, Cocks N, Mobbs E, Hutchins P, McHugh K, de Silva M (1994) Specific learning disability in children with neurofibromatosis type 1: significance of MRI abnormalities. Neurology 44:878-883

16. DiPaolo DP, Zimmerman RA, Rorke LB, Zackai EH, Bilaniuk LT, Yachnis AT (1995) Neurofibromatosis type 1: pathologic substrate of high-signal-intensity foci in the brain. Radiology 195:721-724

17. Denckla MB, Hofman K, Mazzocco MM, Melhem E, Reiss AL, Bryan RN, Harris EL, Lee J, Cox CS, Schuerholz LJ (1996) Relationship between T2-weighted hyperintensities (unidentified bright objects) and lower IQs in children with neurofibromatosis1. Am J Med Genet 67:98-102

18. Szudek J, Evans DG, Friedman JM (2003) Patterns of associations of clinical features in neurofibromatosis 1 (NF1). Hum Genet 112:289-297

19. Gutmann DH, Loehr A, Zhang Y, Kim J, Henkemeyer M, Cashen A (1999) Haploinsufficiency for the neurofibromatosis 1 (NF1) tumor suppressor results in increased astrocyte proliferation. Oncogene 18:4450-4459

20. Listernick R, Ferner RE, Liu GT, Gutmann DH (2007) Optic pathway gliomas in neurofibromatosis-1: controversies and recommendations. Ann Neurol 61:189-198 